Electrochemically assembled quasi-periodic quantum dot arrays

This article has been downloaded from IOPscience. Please scroll down to see the full text article. 1996 Nanotechnology 7360

(http://iopscience.iop.org/0957-4484/7/4/010)

View the table of contents for this issue, or go to the journal homepage for more

Download details:

IP Address: 128.206.162.204

The article was downloaded on $04 / 08 / 2010$ at $21: 13$

Please note that terms and conditions apply. 


\title{
Electrochemically assembled quasi-periodic quantum dot arrays
}

\author{
S Bandyopadhyay $\dagger$, A E Miller $\ddagger$, H C Chang $\ddagger$, G Banerjee $\ddagger$, \\ V Yuzhakov $\ddagger$, D-F Yue $\S$, R E Ricker\|, S Jones $\|$, J A Eastman $\uparrow$, \\ $\mathrm{E} \mathrm{Baugher}^{+}$and M Chandrasekhar ${ }^{+}$
}

\author{
$\dagger$ Department of Electrical Engineering, University of Nebraska, Lincoln, Nebraska \\ 68588-0511, USA \\ $\ddagger$ Department of Chemical Engineering, University of Notre Dame, Notre Dame, \\ Indiana 46566, USA \\ § SGS-Thomson Microelectronics, Inc., Carrolton, Texas 75006, USA \\ || Materials Science and Engineering Laboratory, National Institute of Standards \\ and Technology, Gaithersburg, Maryland 20899, USA \\ q Argonne National Laboratory, Argonne, Illinois 60439, USA \\ + Department of Physics, University of Missouri-Columbia, Columbia, Missouri \\ 65211, USA
}

\begin{abstract}
We describe two electrochemical self-assembly processes for producing highly ordered quasi-periodic arrays of quantum dots on a surface. The advantages of these techniques are: (i) they are 'gentle' and do not cause radiation damage to nanostructures unlike beam lithography, (ii) they have high throughput and are amenable to mass production unlike direct-write lithography, (iii) structures can be delineated on non-planar substrates, and (iv) the techniques are potentially orders of magnitude cheaper to implement than conventional nanosynthesis. Samples produced by these techniques have been characterized by microscopy, optical and transport measurements, Auger and x-ray. These measurements reveal intriguing properties of the nanostructures. In this paper, we describe our initial results and show the promise of such techniques for low-cost and high-yield nanosynthesis.
\end{abstract}

\section{Introduction}

It is well known that conventional fine line lithography (electron beam, focused ion beam, x-ray, STM/AFM etc.) has at least one of two major shortcomings: (i) process related damages incurred through exposure to high-energy beams during pattern delineation (writing) [1-4] or through reactive ion etching (pattern transfer) [5], and (ii) slow throughput associated with direct-write lithography where each wafer is patterned serially one at a time. Recently, parallel electron-beam columns and arrayed nanoprobes have been implemented to increase the throughput and introduce some parallelism into the process, but the cost of such systems is usually prohibitive [6].

In an effort to mitigate these problems, we are developing two electrochemical self-assembly techniques for nanosynthesis that fine-tune a current 'low technology' process routinely available in the anodizing industry into a 'high technology' process for mass-fabricating dense $\left(>10^{12} \mathrm{~cm}^{-2}\right)$ two-dimensional quasi-periodic arrays of metallic, semiconducting or superconducting quantum dots. Samples produced by these techniques have been directly imaged with transmission electron microscopy (TEM), field emission scanning electron microscopy (FESEM) and atomic force microscopy (AFM). Quantitative image analysis carried out by an image capture and digitization system indicates that the average diameter of dots produced by one of these techniques can be as small as 10 $\mathrm{nm}$ with a $1 \mathrm{~nm}$ variation across a wafer, while the average interdot separation can be as small as $40 \mathrm{~nm}$ with a $2 \mathrm{~nm}$ variation. This tight size control is comparable with that obtained with advanced lithographic techniques [7], or molecular-beam epitaxy (MBE) growth of randomly distributed self-assembled quantum dots realized through coherent islanding in Stranski-Krastanow mode [8]. The quantum dots produced by the electrochemical technique have also been characterized by energy-dispersive analysis of x-ray, AC susceptometry, photoluminescence, Raman spectroscopy, absorption and reflectance studies, ellipsometry, and transport studies to investigate their magnetic, optical, electronic and superconducting properties. In section 4 , we describe some of these results; but first we describe the fabrication processes.

\section{Self-assembly of a mask for a periodic array of quantum dots: electropolishing of aluminum}

In this section, we describe the first of the two electrochemical processes that creates a self-assembled mask for a highly periodic array of quantum dots. The array 


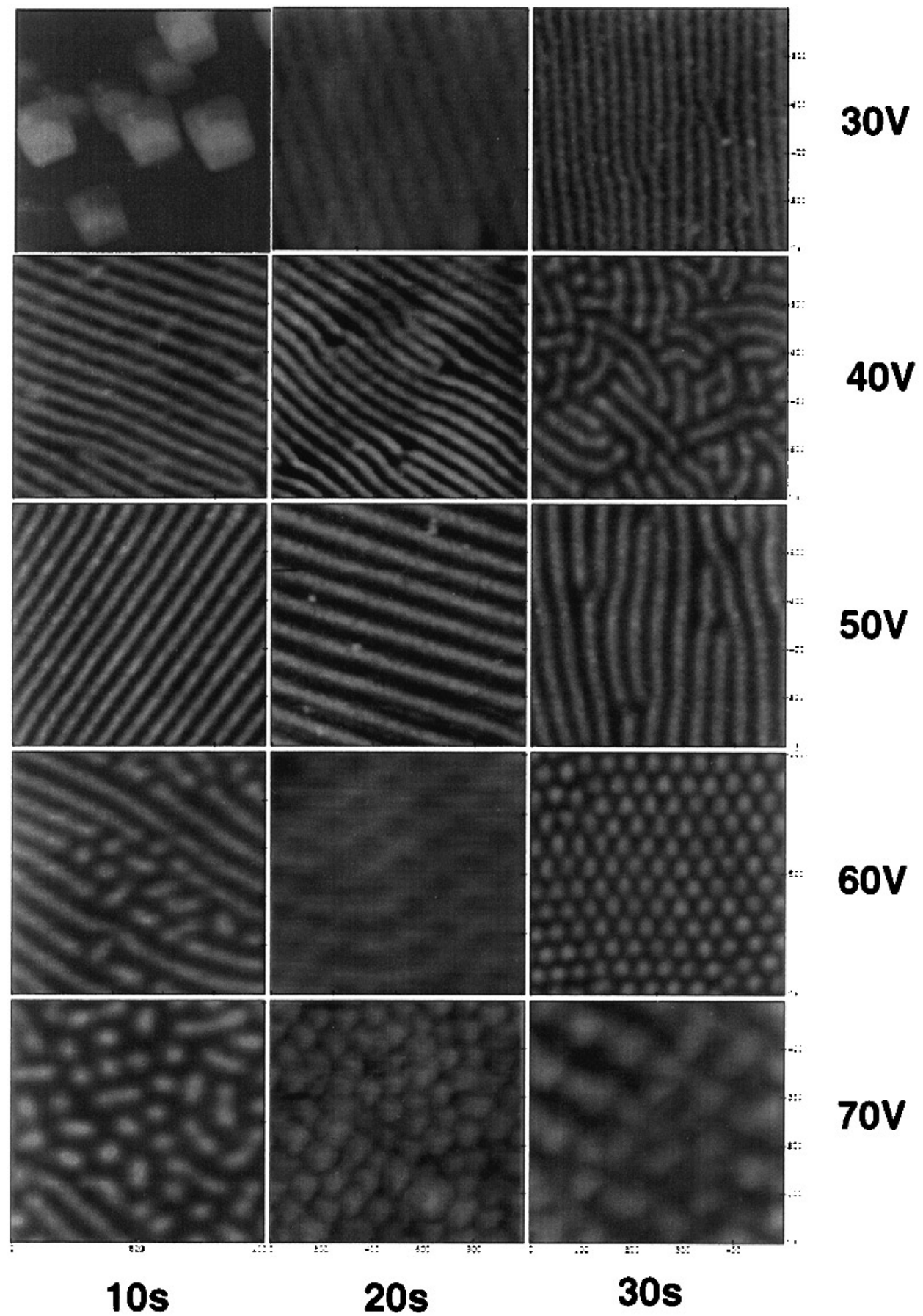

Figure 1. Atomic force micrographs of various patterns that are formed on the surface of electropolished aluminum films after electropolishing in perchloric acid, butyl cellusolve, ethanol and water for various durations of time and at various voltages.

has a hexagonal order (a two-dimensional Fourier transform of an atomic force micrograph of the pattern shows six disinct peaks attesting to the high degree of regimentation and the hexagonal order).

To create this structure, we start with a $99.99 \%$ pure Al film which could be evaporated on any chosen conducting substrate such as doped silicon. This film is then electropolished in a solution of $62 \mathrm{cc}$ perchloric acid, $700 \mathrm{cc}$ ethanol, $100 \mathrm{cc}$ butyl cellusolve and $137 \mathrm{cc}$ distilled water for various time durations and at various voltages. The electropolishing process patterns the surface of the aluminum film into a dimpled and undulating landscape which may or may not have spatial ordering depending on the duration and voltage of electropolishing. Atomic force micrographs (raw data) are shown in figure 1 indicating the patterns that form at various voltages and for various durations of electropolishing. The patterns evolve from a random geography through highly ordered stripes $(50 \mathrm{~V}, 10 \mathrm{~s})$ and an 'egg-carton' pattern $(60 \mathrm{~V}$, 30 s). Obviously, the stripes and the egg-carton are 


\section{TWO TYPICAL ELECTROPOLISHED SURFACE STRUCTURES}
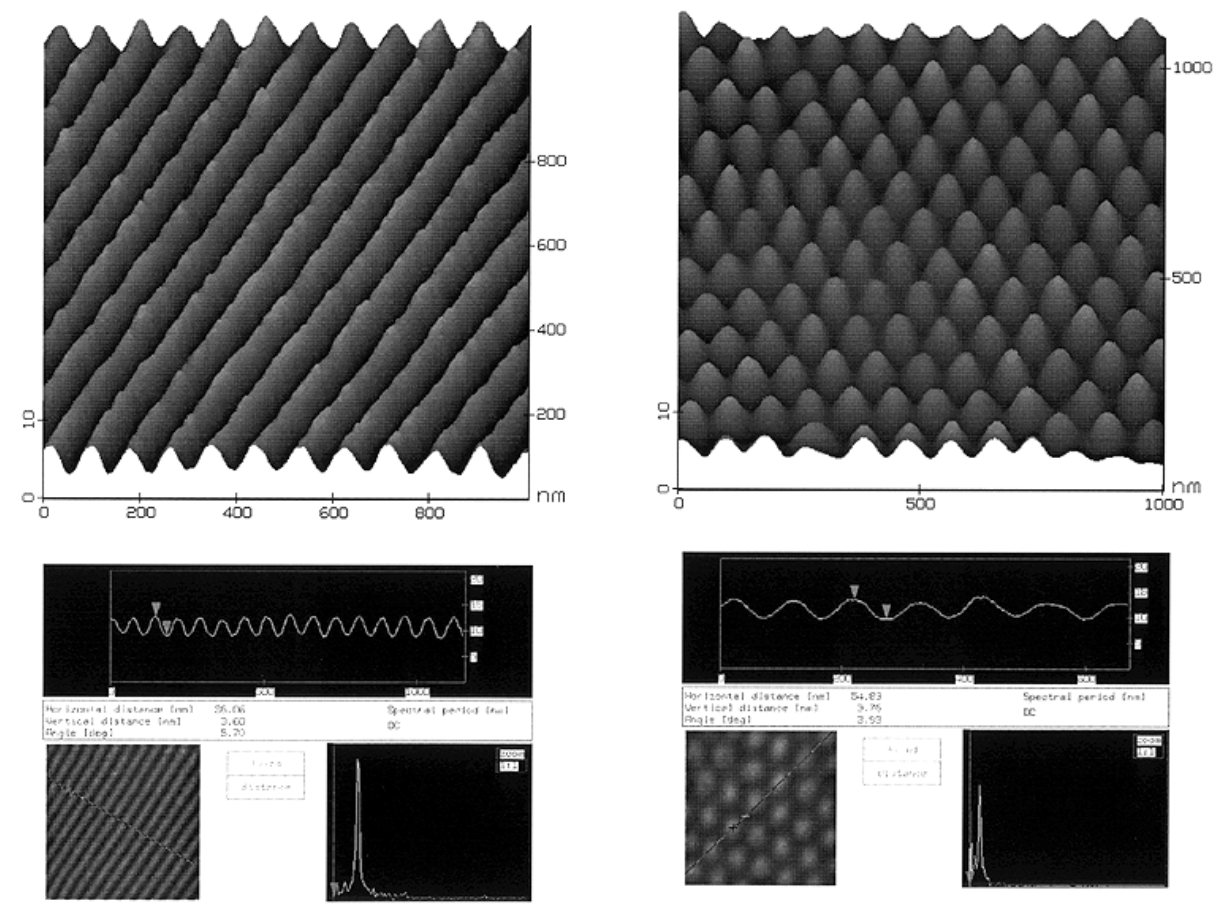

Figure 2. Magnified atomic force micrographs (raw data) of stripes and 'egg-carton' patterns that form on the surface of electropolished aluminum. Stripes form after electropolishing at $50 \mathrm{~V}$ for $10 \mathrm{~s}$ and egg-cartons form after electropolishing at $60 \mathrm{~V}$ for $30 \mathrm{~s}$. These patterns can be used as self-assembled masks for quantum wires and dots. The figure also shows depth profiles along the lines indicated. Well resolved peaks in the Fourier transform of these profiles attest to the high degree of periodicity and order within the scanned area.

useful for making quantum wires and dots respectively. Figure 2 shows a magnified micrograph of the stripe and dot patterns with depth profiles along arbitrary lines drawn on the surface. Fourier transforms of the depth profiles show very narrow and well resolved peaks attesting to the high degree of periodicity of the structures. These structures are incomparably more periodic and ordered than those produced by coherent islanding in StranskiKrastanow growth [8]. The 'pitch' or 'period' of the pattern is about $100 \mathrm{~nm}$ (peak to peak separation) and the height of a crest above a trough is about $3 \mathrm{~nm}$. The hills appear to be conical in shape with a full width at half maximum of about $30 \mathrm{~nm}$.

These structures can be used as self-assembled masks for producing highly periodic arrays of quantum wires and dots. This is achieved as follows (see figure 3). A thin film of aluminum (about $10 \mathrm{~nm}$ thick) is evaporated on a chosen substrate. After electropolishing to produce the desired landscape (stripes or egg-carton), the troughs are selectively etched away in a suitable etchant (note that the peak to valley ratio, or the ratio of the $\mathrm{Al}$ film thicknesses at the crests and troughs is about 3:2). The etching leaves behind the crests which form isolated islands of metal dots in a periodic pattern on the surface of the semiconductor. These Al islands can be used as a mask for mesa-etching of quantum dots using reactive ion etching. Finally, a layer of a material can be deposited on the surface to electrically connect the quantum dots, if desired. The four steps in the process are depicted in figure 3. Note that electrical connection between the quantum dots can also be established via molecular-wire linked clusters [9-11] deposited between the dots. The latter is an established technology at this time.

\subsection{Theory of pattern formation during electropolishing}

The formation of the various patterns during electropolishing can be explained by field-assisted dissolution of metals. We are able to model and mathematically capture the proper conditions for the formation of the patterns on the Al surface and the spatio temporal scalings. During electropolishing, a double layer is formed on the liquid electrolyte side near the constant-potential metal surface as ions are attracted to it under the action of the electropolishing voltage. This sets up a potential gradient normal to the metalelectrolyte interface. The electrolyte contains organic molecules like ethanol and butyl cellusolve. The potential gradient reduces the threshold for the desorption of the organic molecule and consequently, crests, with higher poten- 


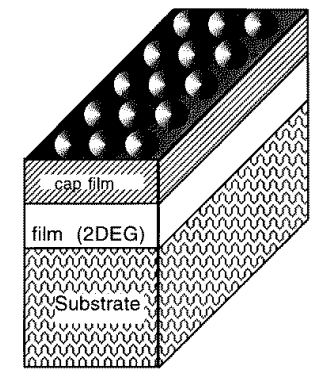

After evaporating Al on the surface and electropolishing

(a)

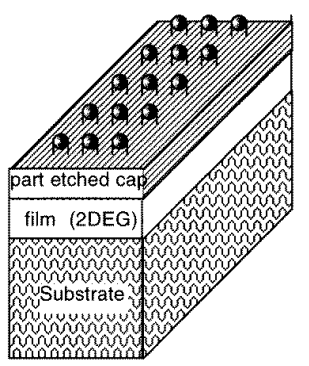

After mesa isolation by etching (only the regions in the film (2DEG) underneath the Al dots are undepleted and form buried quantum dots)

(c)

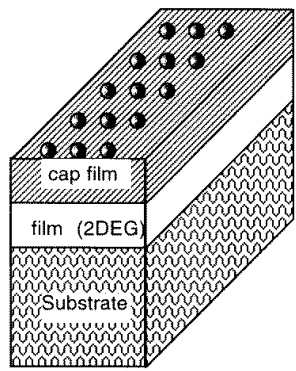

After partially etching the Al

(b)

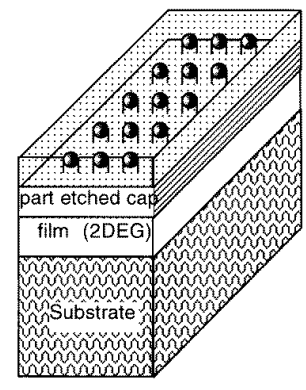

The metallic quantum dots on the surface can be resistively linked by depositing a film on the surface

(d)
Figure 3. Figure indicating the four steps in using the electropolished aluminum as a self-assembled mask to create a periodic array of electrically linked quantum dots on a surface. Such structures have applications in nanoelectronic computing architectures utilizing single electron effects in quantum dots (see [20]). (a). Structure after electropolishing a $100 \mathrm{~nm}$ Al film deposited on a semiconductor layer, $(b)$ after etching the troughs selectively to leave behind the crests that form isolated islands on the surface, $(c)$ shallow mesa etching with reactive ions using the aluminum dots as a mask, $(d)$ electrically connecting the dots by depositing a layer between the mesas.

tial gradients, are preferentially covered by these molecules. The dissolution of $\mathrm{Al}^{3+}$ is then hindered at the crests and troughs dissolve faster (figure 4). This destabilizing mechanism is countered by a curvature stabilizing mechanism which favours surface $\mathrm{Al}$ atoms on a concave surface over dissolved $\mathrm{Al}^{3+}$ ions. As a result, short wavelength disturbances are damped. More importantly, as troughs dissolve deeper, the stabilizing effect at the valley increases while the destabilizing potential gradient in the double layer decreases. Consequently, a finite equilibrium amplitude $h(x, y)$ of the patterns is reached where the two curvaturedependent mechanisms balance to yield regular patterns. The two-dimensional distribution of the amplitude $h(x, y)$ obeys the generalized nonlinear Kuramoto-Sivashinsky equation. Numerical simulation to extract the twodimensional profile of $h(x, y)$ shows patterns that are strikingly similar to the experimentally observed patterns. The simulations show clearly that depending on the voltage and duration of electropolishing, one does indeed get stripes or 'egg-carton' pattern or the random patterns as shown in fig-

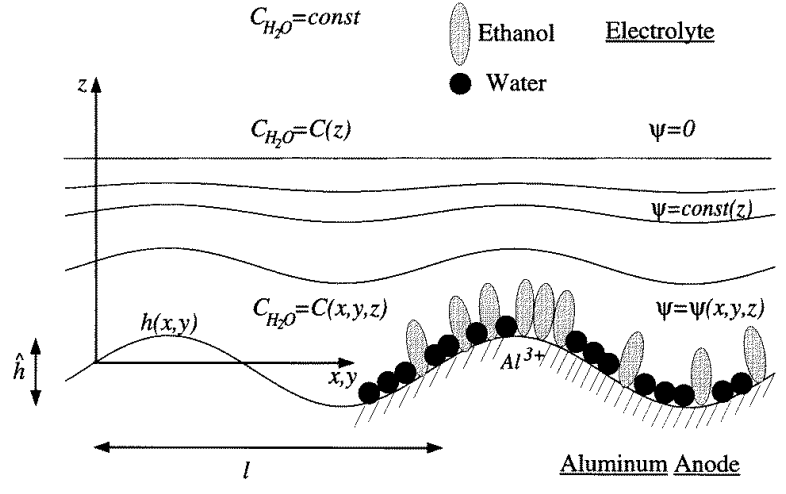

Figure 4. Figure to explain the chemistry of the formation of various surface morphologies on electropolished aluminum. Regions covered by ethanol molecules are protected from dissolution and this results in the formation of various undulating patterns. Ethanol molecules are selectively adsorbed on the crests because the higher potential gradient there reduces the threshold for desorption of ethanol molecules. The constant potential $(\psi)$ surfaces are schematically depicted.

ure 1. Details of these results will be published elsewhere.

We are able to estimate the curvature-dependent threshold for desorption of organic molecules and dissolution of the $\mathrm{Al}$ ion by studying the free energy of the pertinent chemical complexes in the presence of a field. This information allows us to predict the critical voltages beyond which patterns appear, the time scales for transition from stripes to dots, and the characteristic length scales of both patterns. Because of the activated desorption process, the wavelength or 'pitch' of the patterns scales as the exponential of the square of the electropolishing voltage as shown in figure 5 . We also believe that by reducing the temperature of the electrolyte during electropolishing, we can reduce the pitch of the pattern and also the full-widthhalf-maximum of the crests which will result in smaller quantum dots and a denser array. This, however, remains to be tested at this time.

\section{Nanoporous films formed by anodization of aluminum}

We now describe the second process for the creation of quasi-periodic arrays of quantum dots. This technique produces quantum dot arrays with somewhat reduced periodicity, but the individual dot diameter is about five times smaller $(\sim 10 \mathrm{~nm})$. We DC-anodize the electropolished aluminum film produced in the previous step under a constant current density of $40 \mathrm{~mA} \mathrm{~cm}^{-2}$ at room temperature in $15 \% \mathrm{H}_{2} \mathrm{SO}_{4}(\mathrm{pH}=0.6)$. The $\mathrm{Al}$ film is used as the anode and a platinum mesh as the cathode. Anodization creates a porous alumina film on the surface of $\mathrm{Al}$ with a regimented, hexagonal close-packed arrangement of nanopores as shown in the idealized diagram in figure 6 . Formation of this film has been known in the anodizing industry for the last 50 years and has been recently revisited and studied by a number of researchers [12-15]. A bright field transmission electron micrograph of an actual porous alumina film is shown in figure 7. Image analysis of such 


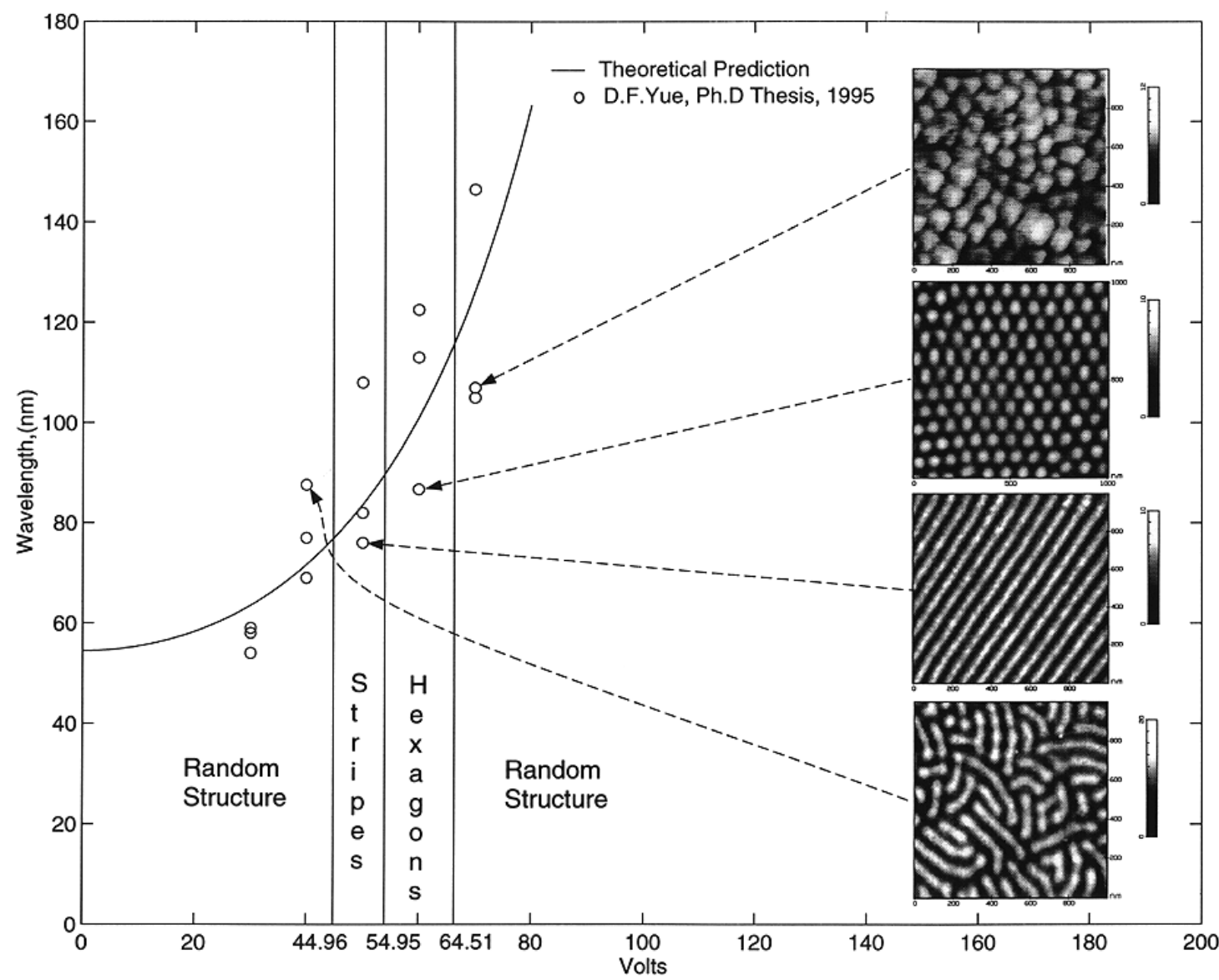

Figure 5. Dependence of the wavelength (or pitch) of the periodic patterns on electropolishing voltages. The solid curve is the computed theoretical result and the experimental data are due to one of us (D-F Yue).

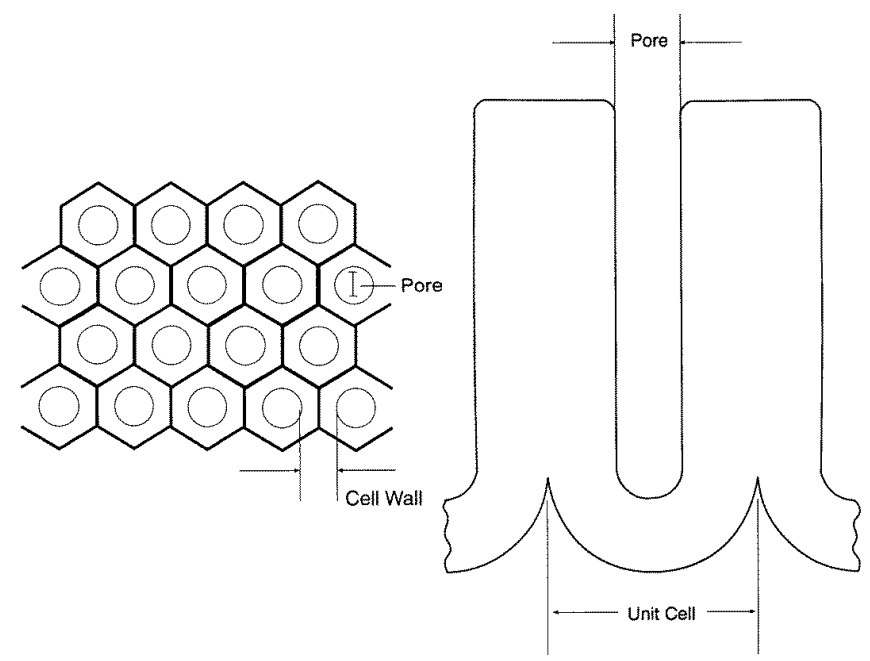

Figure 6. Schematic depiction of the nanoporous film that forms on the surface of aluminum upon anodization. The pores have a hexagonal order. Both the top view and the side view of a pore are shown.

micrographs reveals that typically the average diameter of the pores is $\sim 10 \mathrm{~nm}$ with a $7 \%$ standard deviation and the center-to-center separation is about $40 \mathrm{~nm}$ with a $2 \%$ standard deviation. Histograms of the pore diameter distribution are approximately Gaussian.

Based on extensive TEM, field-emission SEM and
AFM characterization of the nanoporous films formed under various conditions of anodization, we have arrived at the following conclusions.

- Other factors being the same, the pore density decreases and the pore diameter increases slightly with increasing anodizing current density. 


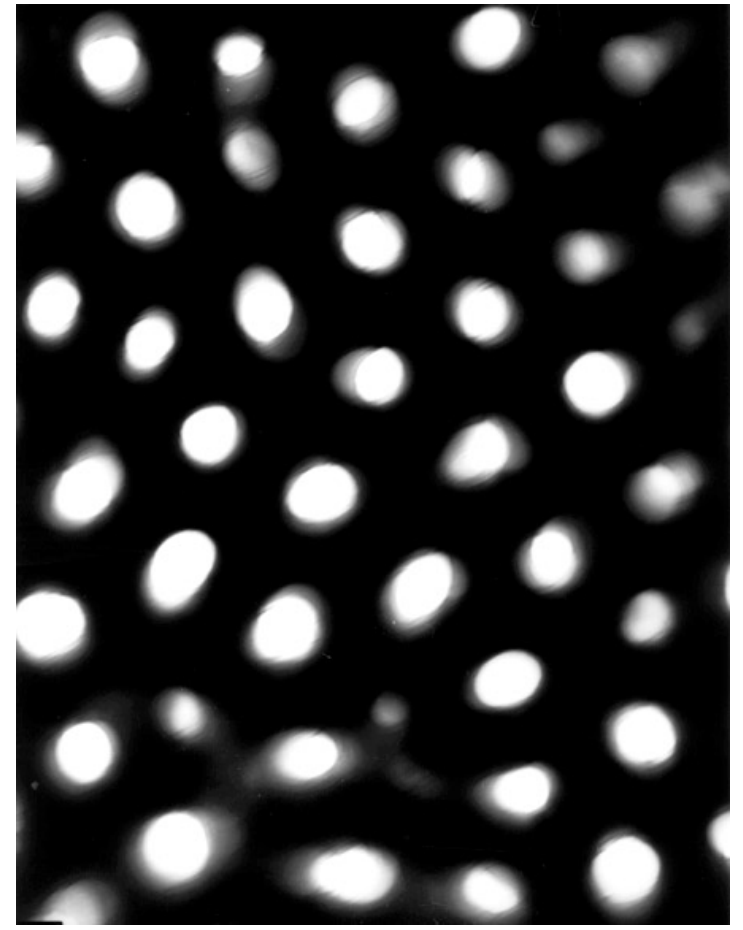

Figure 7. A bright field transmission electron micrograph of a nanoporous film formed by anodizing aluminum in 15\% sulfuric acid at room temperature with a current density of $40 \mathrm{~mA} \mathrm{~cm}^{-2}$. The average pore diameter is $15 \mathrm{~nm}$. The ordering in some regions is clearly hexagonal. TEM samples are produced by dissolving the aluminum backing from the nanoporous film with bromine/methanol and capturing the released porous film in TEM grids.

- The control over pore size, pore density, and most importantly, regimentation (or spatial ordering) improves with increasing current density. Our most regimented pore arrangements are those that are produced at the highest current densities. The highest current densities also result in the minimum standard deviation in the pore diameter, thereby affording the best feature size control.

- Pores tend to nucleate in regions of increased surface elastic energy. Typically grain boundaries are preferred sites as shown in figure 8.

- The pore depth depends strongly on the time of anodization. For the first few seconds of anodization (typically 6-7 s for a current density of $40 \mathrm{~mA} \mathrm{~cm}^{-2}$ ), a barrier layer of alumina forms on the surface and then the pores begin to nucleate. The pore depth continues to increase with increasing duration of anodization. To make pores that are about $10 \mathrm{~nm}$ deep, the time required is usually a few seconds. The pore depth also depends slightly on the underlying crystallographic orientation of the underlying aluminum grain. This is shown clearly in figure 9.

The pore diameter, pore depth, pore density and regimentation can be controlled by fine tuning the temperature, the $\mathrm{pH}$ of the acid, the chemical composition of the acid used during anodization (generally $\mathrm{H}_{2} \mathrm{SO}_{4}$ gives the smallest pore diameter while phosphoric and

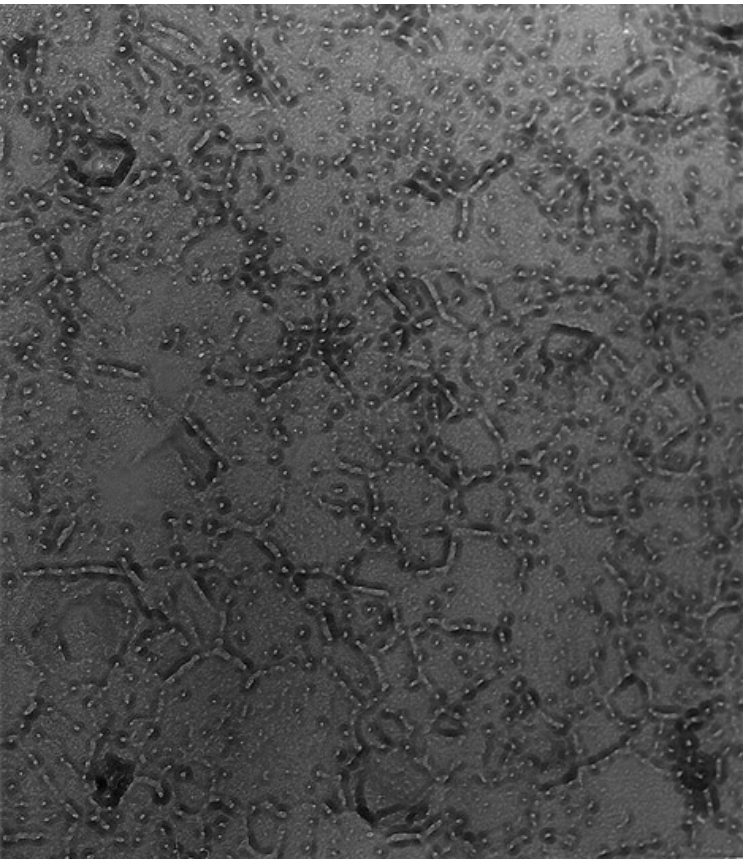

Figure 8. A bright field transmission electron micrograph of pores nucleating on a $300 \mathrm{~nm}$ thick layer of Al evaporated on a single crystal silicon substrate. The evaporated layer was composed of ultrafine grain aluminum with typical grain diameters of $1 \mu \mathrm{m}$. The anodization was carried out in $15 \%$ sulfuric acid with a current density of $30 \mathrm{~mA} \mathrm{~cm}^{-2}$. The magnification is $90000 \times$. Note that pores begin to nucleate at first along grain boundaries which are regions of increased surface elastic energy.

oxalic acids produce much larger pore diameters), and the current density. Pore diameters as small as $5 \mathrm{~nm}$ have been reported in the literature [15]. In some situations, quite well regimented arrays of pores can be produced as shown by the transmission electron micrograph in figure 10. The pore densities in such structures can approach $10^{12} \mathrm{~cm}^{-2}$ which is excellent for applications in high density magnetic storage or high density semiconductor dot arrays for nonlinear optical devices. If the periodicity can be improved slightly, these will also result in self-organized two-dimensional dielectric structures that exhibit a photonic bandgap for all electromagnetic wave propagation directions along the plane of the film [16]. The bandgaps will open up at wavelengths corresponding to deep ultraviolet or possibly $\mathrm{x}$-ray.

\subsection{Theory of pore formation}

The mechanism of pore formation is not entirely different from that which undergirds the formation of the undulating patterns during electropolishing. In contrast to electropolishing, where the potential gradient occurs within the double layer on the metal surface, the potential gradient during anodization lies mainly in the oxide layer which is insulating. Dissolution occurs because the surface oxygen atoms prefer to be surrounded by water molecules in the bulk solution owing to the presence of the electric field. Pitting initiates at a thinner part of the oxide layer because 


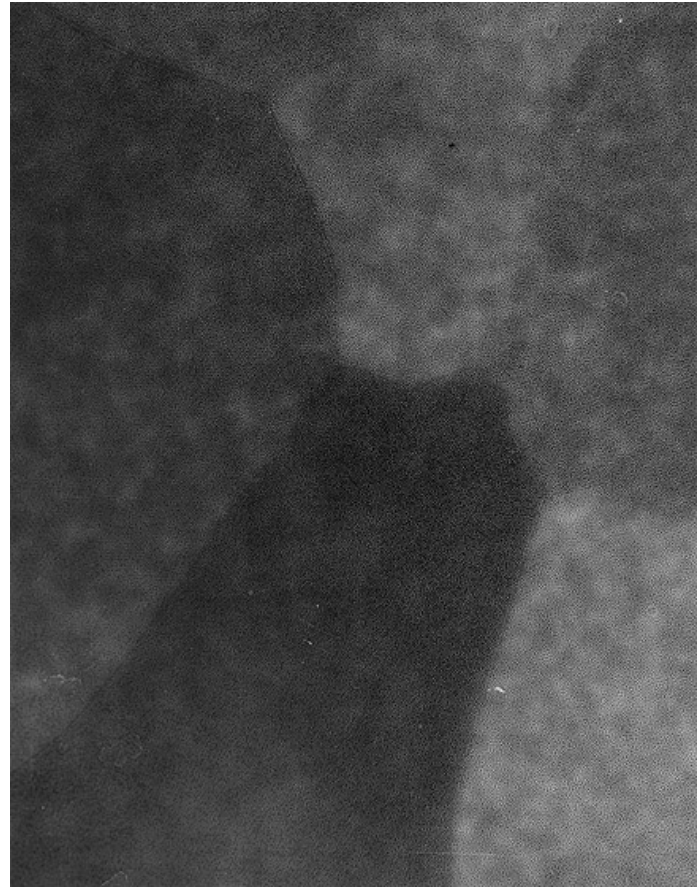

Figure 9. A bright field TEM image of pores formed on various grains in a large grained polycrystalline Al substrate anodized in sulfuric acid at room temperature for $6 \mathrm{~s}$ with a constant current density of $30 \mathrm{~mA} \mathrm{~cm}$. The magnification is $12000 \times$. Some (but not all) grain boundaries are decorated with extremely regimented arrays of pores indicating once again that regions of increased elastic energy produce better regimentation. Lighter shades indicate smaller film thickness (more transparent to transmission electron beam). Thus, the thickness of the anodized film depends somewhat on the crystallographic orientation of the underlying aluminum.

of the enhanced electric field there. This accelerates the solvation rate of the surface oxygen atoms. Since the potential gradient increases as the oxide layer thins, the pits (or pores) grow self-catalytically because of the positive feedback mechanism. The curvature-reduced dissolution rate serves only to select the size of the pore. Owing to the fact that the potential gradient scales linearly with the oxide layer thickness, the pore separation and the pore diameter scale linearly with the anodizing voltage - a drastically different situation from the exponential scaling associated with electropolishing. Because the oxide layer dissolution is determined almost solely by its thickness, these pore size scalings are universal correlations independent of the electrolyte composition (see figure 11). The basic mechanism is, however, somewhat complicated by the field driven flux of oxygen ions through the oxide (alumina) film which thickens the layer. The mechanism of pore initiation is schematically depicted in figure $12(a)$. As shown from our numerical construction of the pit/pore in figure 12(b), the enhanced potential gradient at a pit also produces a thick oxide layer which obviously stabilizes further growth. This secondary mechanism finally arrests the accelerated pore growth and determines the final pore depth. Unlike the pore diameter and the interpore separation, the scaling of the pore depth with the applied voltage is a function of

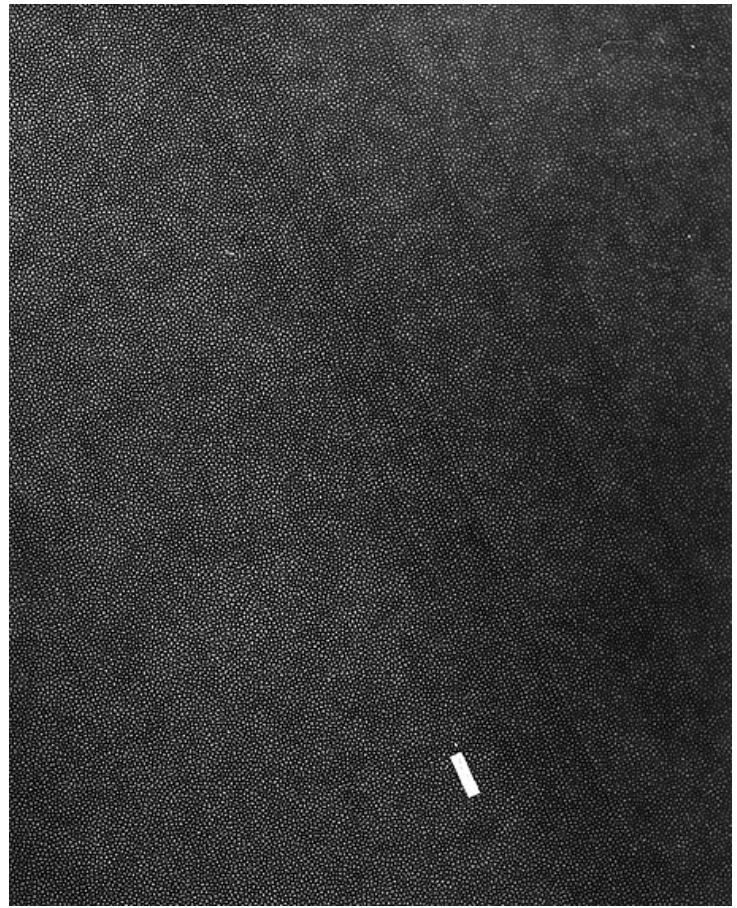

Figure 10. A bright field TEM image of pores taken at a low magnification of $30000 \times$. The white bar is $300 \mathrm{~nm}$. The anodizing current density was $40 \mathrm{~mA} \mathrm{~cm}{ }^{-2}$ and the aluminum film was cold worked. The image shows fairly long range regimentation along strips oriented in the direction of the white bar. These strips are several microns long and few hundreds of $\mathrm{nm}$ wide. Adjacent strips are separated by regions of short range regimentation. The pore density is $4 \times 10^{10} \mathrm{~cm}^{-2}$ which is excellent for high density magnetic storage or semiconductor quantum dot arrays for applications in nonlinear optics.

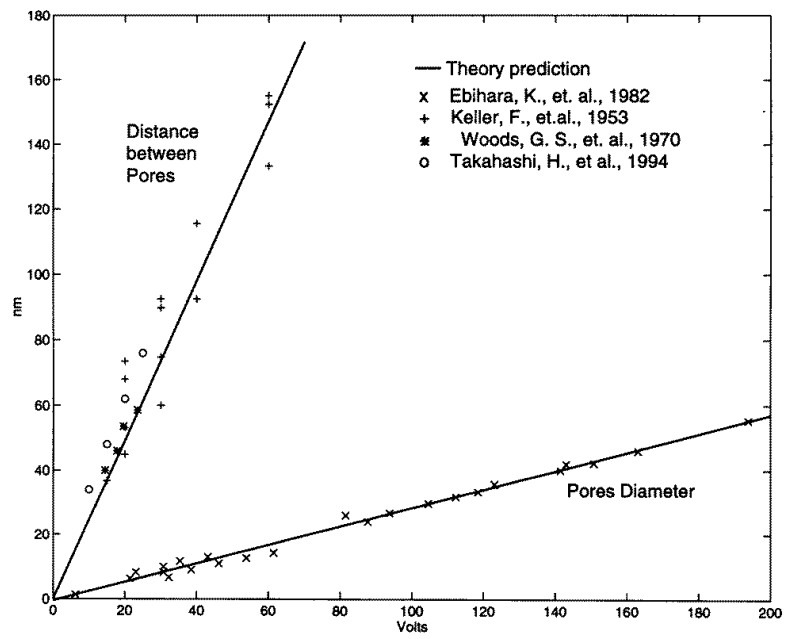

Figure 11. Dependence of the pore diameter and interpore separation on the anodizing voltage. The solid lines are the computed theoretical results and the experimental data are those reported by various researchers.

the electrolyte composition, especially the concentration of oxidizing ions.

This model of pore formation should be compared and contrasted with the various models proposed for the 


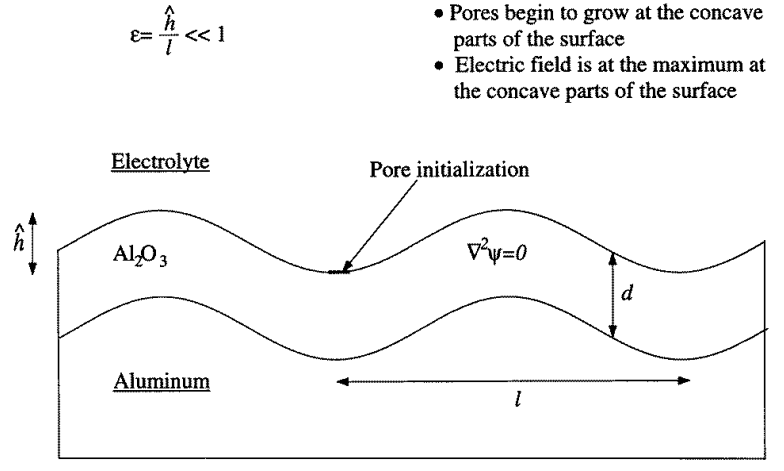

(a)

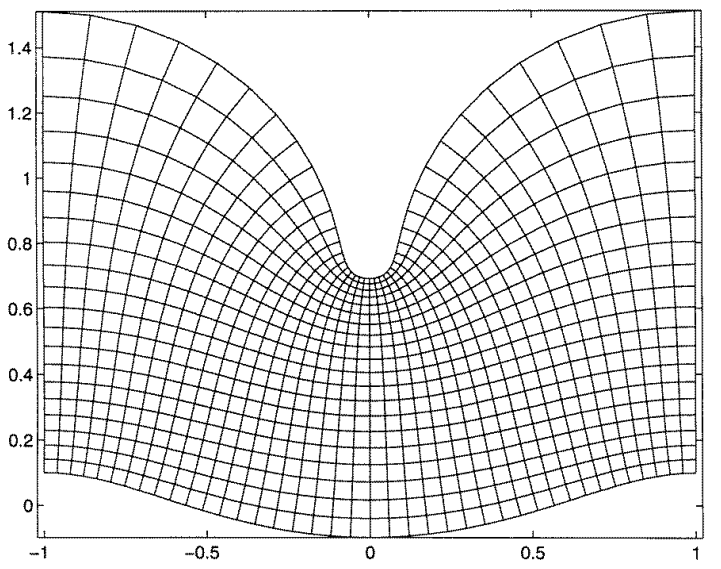

(b)

Figure 12. (a) Schematic representation of the initiation of pore nucleation. (b) Numerical construction of a pore showing its formation.

formation of porous silicon [17].

\subsection{Filling the pores by electrodeposition}

To finally create 'quantum dots', the nanopores formed by anodization are selectively filled with the material of interest by electrodeposition. When this is complete, one obtains a two-dimensional quasi-periodic arrangement of quantum dots (or short, vertical quantum wires if the pore depth significantly exceeds the pore diameter) surrounded by $\mathrm{Al}_{2} \mathrm{O}_{3}$ (a dielectric insulator). The $\mathrm{Al}_{2} \mathrm{O}_{3}$ insulator can be removed, if desired, by first depositing a suitable film on the filled pores (third step) and then dissolving away the insulator in an etching solution (final step). This produces a quasi-periodic quantum dot array with the film deposited in the third step acting as the substrate (see figure 13). The last two steps may be unnecessary in most electronic, magnetic and optical applications since the presence of an insulating dielectric surrounding the quantum dots does not interfere with these applications. We describe the basic pore-filling step below.

The pores are filled with the material of interest by electrodeposition. For this, the porous film is first washed in distilled water and dried. The pores are enlarged and 'rounded', if needed, by dissolving part of the surrounding

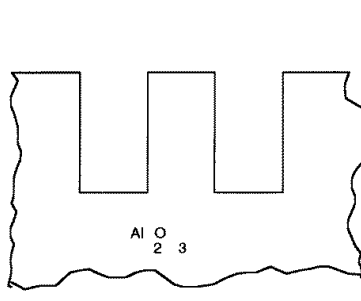

(a)

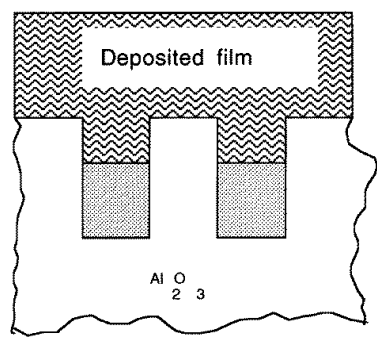

(c)

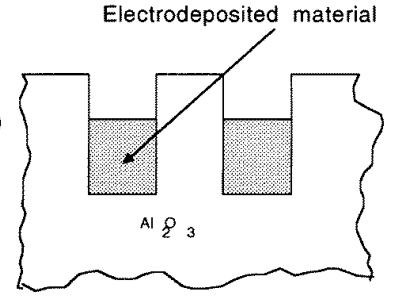

(b)

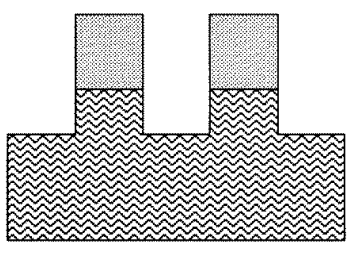

(d)
Figure 13. Various steps in producing quantum dots by electrodepositing materials of interest within pores. (a) Side view of the nanoporous film, $(b)$ after electrodepositing the material of interest within the pores, (c) depositing a film over the structure, $(d)$ selectively etching away the alumina to create mesa quantum dots on the film deposited in the third step acting as the final substrate.

alumina walls in an aqueous acid solution. After this, the film is washed to remove debris and dried.

For electrodepositing most metals (e.g. $\mathrm{Fe}, \mathrm{Ni}, \mathrm{Pb}$, etc.), the porous film is placed in an acidic, non-cyanide bath that does not attack alumina, and AC current is passed at a suitable rms voltage between 18 and $20 \mathrm{~V}$ using the bottom $\mathrm{Al}$ substrate and a graphite plate immersed in the bath (or a platinum mesh) as the electrodes. The bath contains a soluble salt of the metal which ionizes to produce the metal ion which is preferentially electrodeposited within the pores since the pores offer the least impedance paths between the two electrodes. The time of electrodeposition determines the depth of pore filling. To control the filling factor (how much of the pore is filled up), one can monitor the surface resistivity of the film or carry out real-time impedance spectroscopy. The surface resistivity correlates very well with the filling factor and can be used to calibrate this process. We show a cross-sectional transmission electron micrograph of nickel filled pores in figure 14. These TEM specimens were produced by ultramicrotomy. For a $5 \mathrm{~s}$ electrodeposition, only a small part of the pores is filled resulting in $10 \mathrm{~nm}$ sized quantum dots of nickel situated at the bottom of the pores (figure 14(a)). For a $20 \mathrm{~s}$ electrodeposition, the pores are filled up almost to the brim resulting in short and vertical quantum wires of nickel (200 $\mathrm{nm}$ height) within the pores (figure 14(b)).

For electrodepositing compound semiconductors like $\mathrm{CdS}$ or $\mathrm{ZnS}$ within the pores, the alumina film is first immersed in $\mathrm{H}_{2} \mathrm{SO}_{4}$ and $\mathrm{AC}$ electrolysis is performed for $10 \mathrm{~s}$ with a rms current density of $40 \mathrm{~mA} \mathrm{~cm} \mathrm{~cm}^{-2}$ which leaves behind the $\mathrm{S}^{2-}$ ion in the pores. This is followed by immersing the film in boiling deionized water containing a $10 \%$ solution of $\mathrm{CdSO}_{4}$ or $\mathrm{ZnSO}_{4}$. This solution must be 


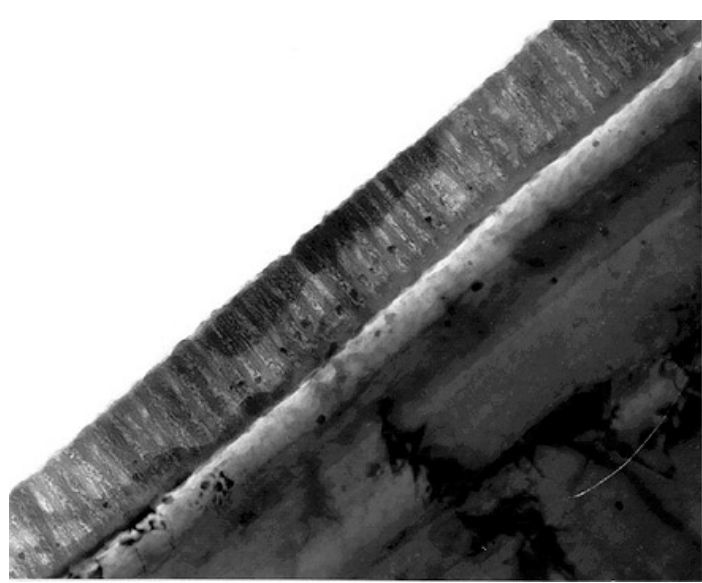

(a)

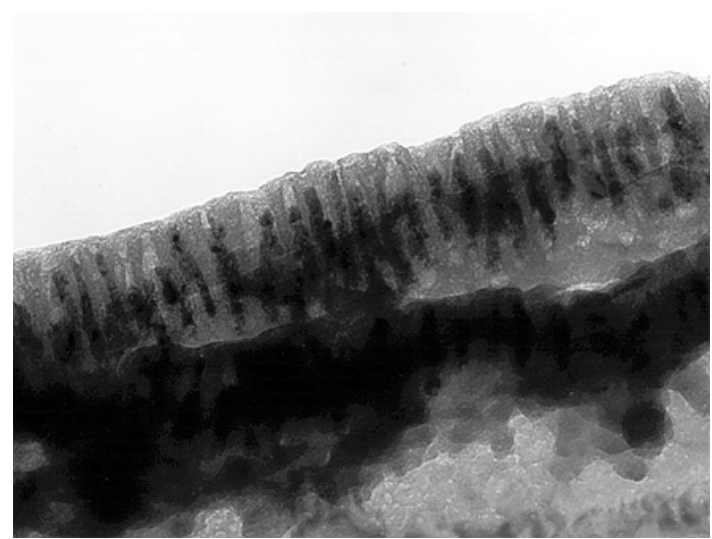

(b)

Figure 14. A cross-section TEM image of nickel filled pores. (a) After electrodepositing nickel for $5 \mathrm{~s}$, and $(b)$ after electrodepositing nickel for $20 \mathrm{~s}$. In the first case, we have obtained spherical nickel quantum dots of $10 \mathrm{~nm}$ diameter and in the second case, we have obtained cylindrical short quantum wires with $10 \mathrm{~nm}$ diameter and $200 \mathrm{~nm}$ height.

chemically pure. Quite often, this is the major source of contamination. The cation $\mathrm{Cd}^{2+}$ or $\mathrm{Zn}^{2+}$ in the solution reacts with the $\mathrm{S}^{2-}$ in the pores to form $\mathrm{CdS}$ or $\mathrm{ZnS}$ inside the pores. The time of immersion determines the amount of compound produced by this reaction and therefore determines the depth of pore filling. Usually not all the $S^{2-}$ reacts with the metal ion so that some excess $S^{2-}$ is left behind in the pores. This does not cause a problem. Deposition of $\mathrm{CdS}$ or $\mathrm{ZnS}$ takes place within the pores at a slow rate thereby affording excellent control. The bath can be used for several successive operations if material purity is not a serious concern. The water for rinsing is treated by usual chemical treatment or ion exchange before being drained.

The materials electrodeposited within the pores may not be crystalline. However, crystallinity may be restored, at least partially, by thermal annealing following the deposition. For $\mathrm{CdS}$, we anneal at $150{ }^{\circ} \mathrm{C}$ for $6-24 \mathrm{~h}$.

The above process can be used to create quantum dots of ZnTe, CdTe, ZnSe, CdTe, GaAs, InAs, InP, GaP, etc. For tellurides, one uses telluric acid instead of sulfuric acid; for arsenides, one uses arsenic acid, for phosphides, one uses phosphoric acid and for selenides, one uses selenic acid.

After the electrodeposition step, one is left with a quasi-periodic arrangement of quantum dots surrounded by $\mathrm{Al}_{2} \mathrm{O}_{3}$. The oxide can be removed, if desired, by following the last two steps illustrated in figure 13. Usually however, this is unnecessary.

Before concluding this section, we would like to point out a few advantages of this technique for producing quasiperiodic quantum dot arrays:

(1) It is a 'gentle' electrochemical technique and does not subject the nanostructures to high-energy beams such as x-ray, electron or ion beam. There is no etching step which would have caused material damage. Consequently, it may result in better material quality when perfected.

(2) It is amenable to mass production. The number of wafers that can be handled simultaneously is limited by the size of the anodizing or electrodeposition bath. For a sufficiently large bath, this number can be several millions.

(3) One can produce nanostructures on curved substrates (such as the surface of a cylinder or sphere) rather than plane substrates exclusively. Anodization of curved substrates and the subsequent ac electrodepostion is no different than for plane surfaces.

(4) The technique is much cheaper than most other techniques for nanosynthesis. The capital investment cost is a few thousand dollars rather than a few million dollars.

\section{Characterization}

We now present some results that characterize the properties of the quantum dots. We chose six different methods for studying the dots.

(1) Analysis of energy dispersive x-ray;

(2) optical characterization such as photoluminescence, absorption and reflectance, ellipsometry and Raman spectroscopy;

(3) magnetic and transport studies;

(4) cross-sectional TEM;

(5) AC susceptometry;

(6) Scanning Auger spectroscopy.

We will discuss some of them here.

\subsection{Energy-dispersive analysis of $x$-ray scattering}

Energy-dispersive analysis confirms the presence of the material of interest within the pores. The data for $\mathrm{Pb}$ quantum dots produced by this technique are shown in figure 15 . 


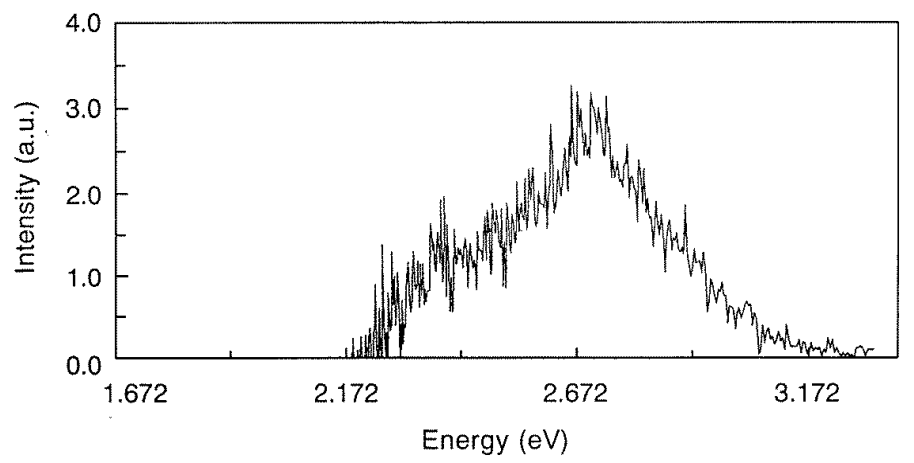

Figure 16. Photoluminescence spectrum of CdS quantum dots prepared by electrodepositing CdS within the pores. The peak shows a blue shift of $200 \mathrm{meV}$ from the bulk bandedge indicating quantum confinement. The calculated dot diameter is $6 \mathrm{~nm}$ which is less than the TEM observed pore diameter of $13 \mathrm{~nm}$. The difference of $7 \mathrm{~nm}$ can be explained by side depletion by interface states, or by the fact that the blue shift is not entirely due to quantum confinement, but partly due to strain. The large full-width-at-half-maximum could be due to lack of complete crystallinity, homogeneous and inhomogeneous broadening, strain, phonon-bottleneck effect, etc.

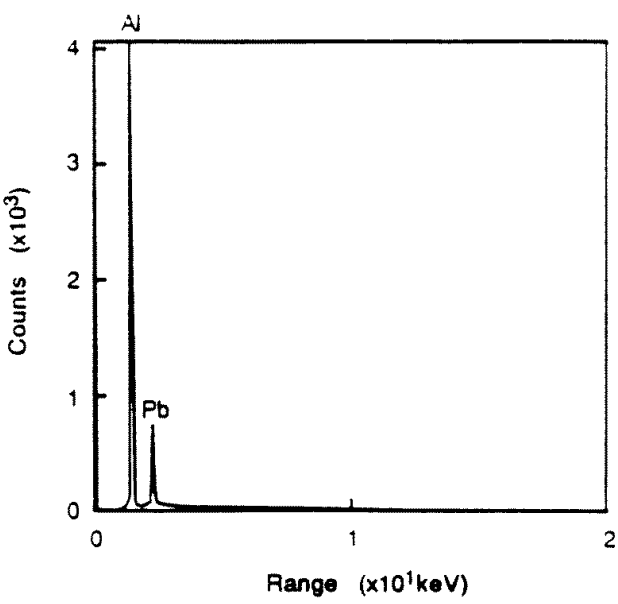

Figure 15. Energy dispersive $x$-ray data showing the presence of $\mathrm{Pb}$ in the pores. The samples were prepared by electrodepositing $\mathrm{Pb}$ into the pores from a solution of lead acetate.

\subsection{Optical characterization}

The photoluminescence data for $\mathrm{CdS}$ dots are shown in figure 16 . Because of the relatively poor quantum efficiency and the dimness of photoluminescence, the spectrum is noisy. However, it shows definite indication of quantum confinement since the peak is blue shifted from the bulk CdS bandedge by $200 \mathrm{meV}$ (bulk CdS bandgap at the measurement temperature of $14 \mathrm{~K}$ is $\sim 2.41 \mathrm{eV}$ ) [18]. If we neglect any strain-induced shift or any Burstein-Moss shift, then this amount of blue shift indicates a quantum dot diameter of $\sim 6 \mathrm{~nm}$ (in this calculation, we assumed that the electron and heavy-hole effective masses retain their bulk values; $m_{e}^{*}=0.21 m_{0}$ and $\left.m_{h h}^{*}=0.8 m_{0}\right)$. This $6 \mathrm{~nm}$ diameter is somewhat less than what quantitative image analysis indicates $\dagger$. The latter indicates that the physical quantum dot diameter is $\sim 13 \pm 1 \mathrm{~nm}$. However, if the side depletion of the dots (caused by Fermi level pinning)

$\dagger$ If we account for any Burstein-Moss shift (which may not be negligible because the total number of states is small in a quantum dot and can be easily filled), then the quantum dot diameter will be larger than $6 \mathrm{~nm}$. is a mere $3.5 \mathrm{~nm}$, then the effective optical dot diameter measured by this technique will be indeed $6 \mathrm{~nm}$. A mere $3.5 \mathrm{~nm}$ side depletion is quite plausible for these systems.

A disturbing feature in the photoluminescence spectrum is its very broad width (400 meV full width at half maximum). There are at least four possible sources for this broadening.

- Homogeneous broadening caused by either impurities, surface states or by the fact that the CdS quantum dots are amorphous and not crystalline. The dots were annealed for six hours at $150{ }^{\circ} \mathrm{C}$ to recrystallize them. However, this may not have been sufficient. We are investigating this possibility using electron diffraction to ascertain the crystallinity (or lack thereof) of the CdS dots. However, the most likely culprit is strain. These dots are severely strained which may cause very large broadening.

- Inhomogenous broadening in the system. We found that even a $2 \mathrm{~nm}$ variation in the effective dot diameter will result in an inhomogeneous broadening of $\sim 150$ $\mathrm{meV}$. This problem is pathological to extreme quantum confined structures [8]. The laser spot size used in exciting the dots has a diameter of $100 \mu \mathrm{m}$ so that $4 \times 10^{6}$ dots are excited simultaneously. In future, we will etch mesas of area $10 \mu \mathrm{m} \times 10 \mu \mathrm{m}$ and excite them individually to reduce the inhomogenoeus broadening.

- Because of selection rules imposed by restricted momentum conservation in quantum dots, scattering of photoexcited carriers from higher subbands to the lowest subband is inhibited. This so-called 'phonon bottleneck' effect [19] can cause broadening of the spectrum since it promotes transitions involving excited states.

- Finally, it is possible that the broadening is caused by nonlinear optical effects. Since the amount of CdS is very small, it is necessary to use intense excitation so that the photoluminescence is detectable. If nonlinear effects are manifested at this excitation power (and they are expected to be), then harmonic generation may cause a wide spectrum. We are planning to study nonlinear effects by direct transmission/absorption 

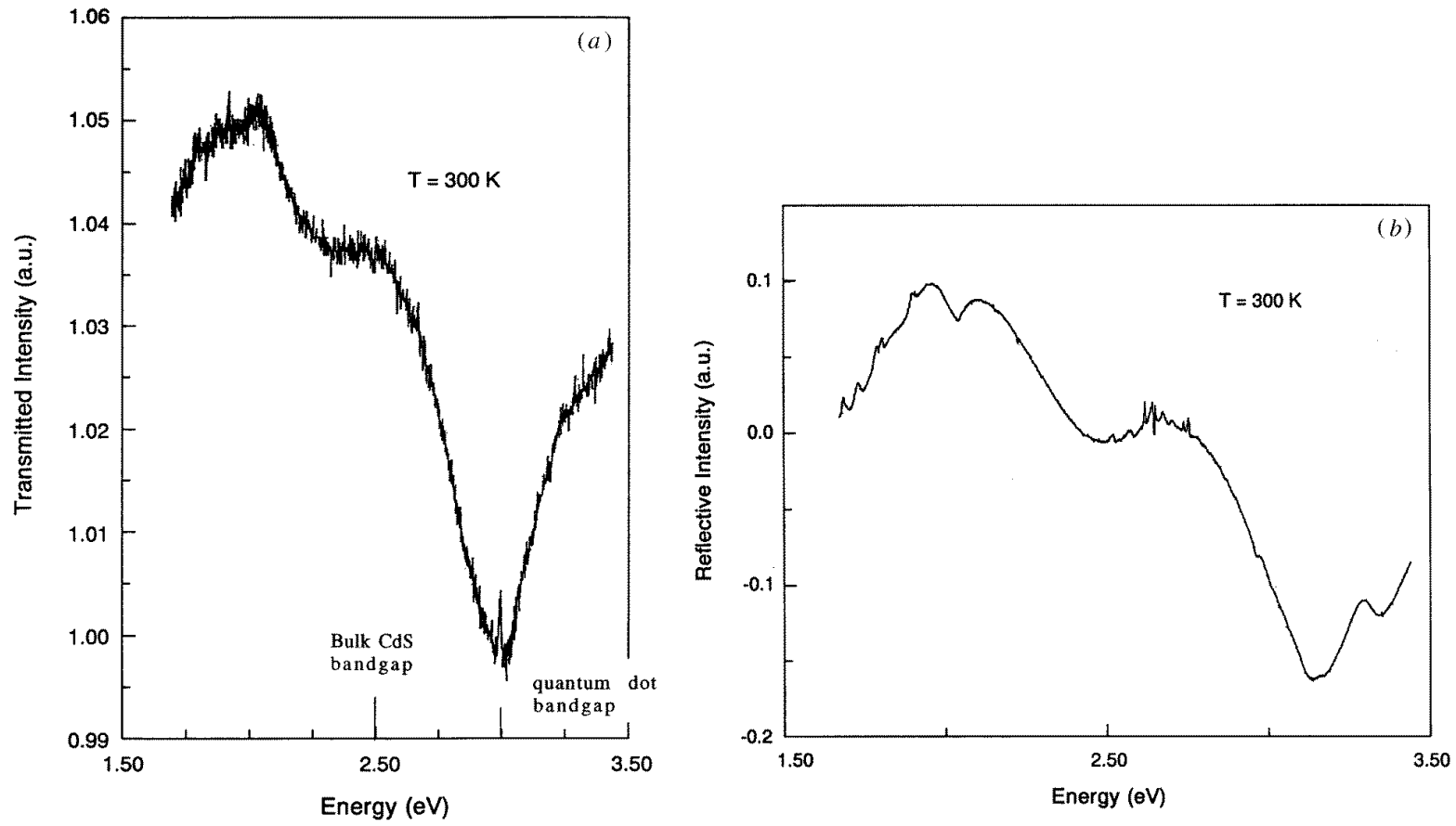

Figure 17. (a) Transmission and (b) reflectance spectrum showing a blue-shifted absorption edge at $3.0 \mathrm{eV}$ which is 500 meV above the bulk bandedge. The calculated diameter of the quantum dots (assuming that the blue shift is entirely due to quantum confinement) is $3.5 \mathrm{~nm}$.

measurements. These measurements will be carried out by stripping the $\mathrm{Al}$ substrate (after the $\mathrm{CdS}$ electrodeposition) using bromine/methanol solution and then capturing the stripped film on a transparent quartz slide. We expect to observe absorption saturation in these dots owing to either phase space filling, or bandgap renormalization, or inherent third order nonlinear susceptibility $\chi^{(3)}$ associated with formation of excitonic complexes (e.g. biexcitons). At the time of writing this manuscript, preliminary evidence of absorption saturation has been observed in pump-probe experiments by our collaborators.

Still smaller structures (produced by shorter duration of electrodeposition) have been characterized by absorption and reflectance studies. The spectra are shown in figure 17. In this case the blue-shift of the absorption bandedge is about $500 \mathrm{meV}$ indicating an effective optical diameter of the dots to be $3.5 \mathrm{~nm}$. These are some of the smallest semiconductor dots produced in non-agglomerated quasiregimented arrays that are optically active.

In addition to photoluminescence, Raman spectroscopy of CdS quantum dots has also been performed. The data are shown in figure 18 where the longitudinal optical (LO) phonon peak for $\mathrm{CdS}$ and a peak at twice the LO frequency are consistently observed. The peaks were observed when the laser photon energy resonantly excited the Raman modes $(2.71 \mathrm{eV}$ or $2.54 \mathrm{eV}$ ) but were not observed when the photon energy was below the bandgap $(2.41 \mathrm{eV})$. These data unambiguously indicate that the material formed within the pores is definitely $\mathrm{CdS}$. Since the peaks were neither significantly wide nor asymmetric in their lineshape, we believe that the $\mathrm{CdS}$ material within the pores is mostly crystalline.

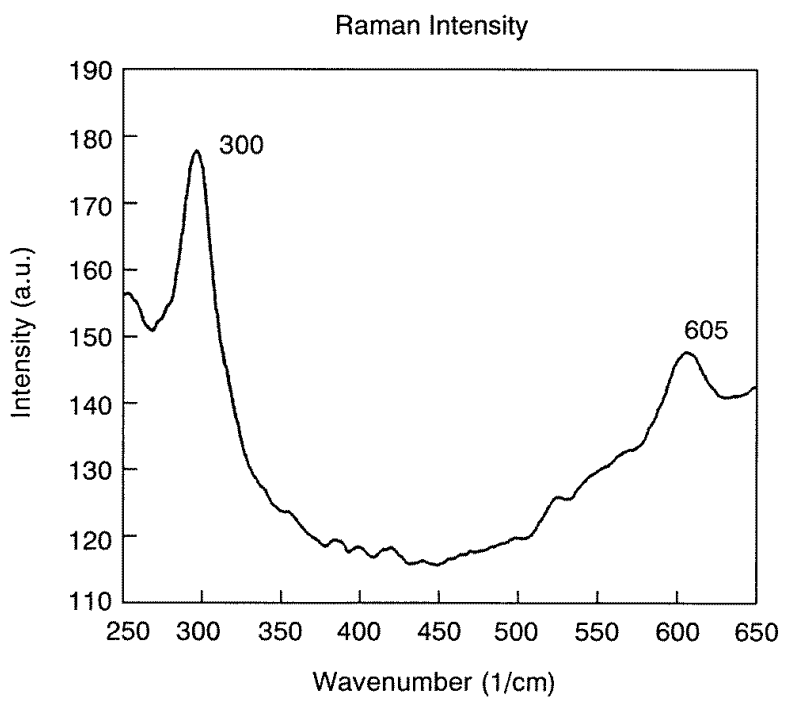

Figure 18. Raman spectra of CdS quantum dots showing the LO and 2LO phonon peaks. Other phonon peaks were not observed possibly because of inadequate quantum efficiency. Observation of these peaks characteristic of CdS indicates the presence of CdS within the pores.

\subsection{Magnetic properties}

To study magnetic properties, we fabricated an array of magnetic quantum dots using the structure shown in figure 14(a). This structure was then electroplated with copper and four leads were attached to the top copper layer. The four terminal resistance of this structure was measured in a magnetic field. Electrons flow in the copper layer but suffer spin-dependent scattering from the magnetic 
moments of the underlying nickel quantum dots. Using a simple model, we predicted that this structure will exhibit giant magnetoresistance. Room temperature measurements indeed showed a $3 \%$ magnetoresistance up to a magnetic field of $2 \mathrm{~T}$ [14].

\subsection{Superconducting properties}

We have deposited the high-temperature superconductor YBCO within the pores from an organic electrolyte. Stoichiometry has been verified by Auger. Microwave surface resistance measurements have shown that these quantum dot superconductors retain the superconductivity and reach zero resistance at a slightly depressed $T_{c}$ of $88 \mathrm{~K}$.

\section{Conclusion}

In conclusion, we have described two electrochemical selfassembly processes that we believe may lead to cheap and versatile techniques for producing regimented quantum dot arrays. Further characterization of the structures and theoretical simulations geared towards understanding the self-assembly mechanism are currently underway.

\section{Acknowledgments}

This work was supported by the Department of Energy grant DE-FG02-90ER45427 administered by the Midwest Superconductivity Consortium (Nebraska, Notre Dame and Missouri), and by the US Army Research Office under grants DAAH04-95-1-0586 (Nebraska and Notre Dame) and DAAL03-92-G0381 and EPSCoR DAAL03-92G0367 (Missouri). The work at Argonne was supported by the US Department of Energy under grant BESDMS-W-31-109-ENG-38. The authors are grateful to Professor Howard Blackstead for microwave measurements of superconducting samples.

\section{References}

[1] Das B, Subramaniam S and Melloch M R 1993 Semiconduc. Sci. Technol. 81347

[2] Tsui D C, Gossard A C and Dolan G J 1983 Appl. Phys. Lett. 42180

[3] Fink T, Smith D D and Braddock W D 1990 IEEE Trans. Electron Devices ED 371422

[4] Stellwag T B, Melloch M R, Cooper J A Jr, Sheppard S T and Nolte D D 1992 J. Appl. Phys. 714509

[5] Knoedler C M 1990 J. Appl. Phys. 681129

[6] Chang T P 1996 Proc. Int. Conf. Quantum Devices and Circuits (Alexandria, Egypt, June 1996) to appear
[7] Sakaki H 1992 Surface Science 267623

Meurer B, Heitmann D and Ploog K 1992 Phys. Rev. Lett. 681371

Wang P D, Sotomayer-Torres C M, Benisty H, Weisbuch C and Beaumont S P 1992 Appl. Phys. Lett. 61946

Schmidt A, Forchel A, Straka J, Gyuro I, Speier P and Zielenski E 1992 J. Vac. Sci. Technol. B 102896

[8] Bawendi M G et al 1990 Phys. Rev. Lett. 651623

Leonard D, Krishnamurty M, Reaves C M, DenBaars S P and Petroff P M 1993 Appl. Phys. Lett. 633203

Moison J M, Houzay F, Barthe F, Leprince L, André E and Vatel O 1994 Appl. Phys. Lett. 64196

Ledentsov N N et al 1995 Proc. Int. Conf. Phys. of Semiconductors ed D J Lockwood (Singapore: World Scientific) pp 1855

Heitz R et al 1996 Appl. Phys. Lett. 68361

Grundmann M 1996 Adv. Solid State Phys. 35123

[9] Andres R P, Bielefeld J D, Henderson J I, Janes D B, Kolagunta V R, Kubiak C P Mahoney and Osifchin R G Science to appear

[10] Andres R P, Bein T, Dorogi M, Feng S, Henderson J I, Kubiak C P, Mahoney W, Osifchin R G and Reifenberger R G 1996 Science 2721323

[11] Andres R P 1994 Conf. on Ordered Molecular and Nanoscale Electronics (Kona, Hawaii, June 1994)

[12] Diggle J W, Downie T C and Goulding C W 1969 Chem. Rev. 69, 365

Huber C A, Huber T E, Sad Mqio, Lubin J A, Manalis S and Prater C B 1994 Science 263800

Moskovits M 1993 US Patent \# 5,202,290, issued April 13

[13] Penner R M and Martin C R 59, 2625

Brumlik C J, Martin C R and Takuda K 1992 Anal. Chem. 641201

Klein J D et al 1993 Chem. Mater. 5902

[14] Bandyopadhyay S, Miller A E and Chandrasekhar M 1995 Proc. SPIE 239711

Miller A E, Yue D-F, Banerjee G, Bandyopadhyay S, Ricker R E, Jones S and Eastman J A 1994 Quantum Confinement: Physics and Applications ed M Cahay et al (Pennington, NJ: The Electrochemical Society Inc.) p 166

Yue D-F, Banerjee G, Miller A E and Bandyopadhyay S 1996 Superlat. Microstruct. 19191

Yue D-F 1995 PhD thesis University of Notre Dame and references therein

[15] Martin C R 1994 Science 2661961

[16] Kazuaki Sakoda 1995 Phys. Rev. B 52, 8992 and references therein

Rosenberg A, Tonucci R J, Lin H B and Shirley E L 1996 Phys. Rev. B 54 R5195

[17] Beale R et al 1985 J. Cryst. Growth 73622

Searson P C 1993 J. Appl. Phys. 72253

Kang Y 1993 J. Electrochem. Soc. 1402258

[18] Sze S M 1981 Physics of Semiconductor Devices 2nd edn (New York: Wiley)

[19] Bockelmann U and Bastard G 1990 Phys. Rev. B 428947

[20] Bandyopadhyay S and Roychowdhury V P 1995 Phys. Low Dimensional Structures 8/929 